\title{
Measurements of neutron cross sections for advanced nuclear energy systems at $\mathbf{n}$ TOF (CERN)
}

M. Barbagallo ${ }^{1, a}$, N. Colonna ${ }^{1}$, S. Altstadt ${ }^{2}$, J. Andrzejewski ${ }^{3}$, L. Audouin ${ }^{4}$, V. Bécares ${ }^{5}$, F. Bečváŕ ${ }^{6}$, F Belloni ${ }^{7}$, E. Berthoumieux ${ }^{7,8}$, J. Billowes ${ }^{9}$, D. Bosnar ${ }^{10}$, M. Brugger $^{8}$, M. Calviani ${ }^{8}$, F. Calviño ${ }^{11}$, D. Cano-Ott ${ }^{5}$, C. Carrapiço ${ }^{12}$, F. Cerutti ${ }^{8}$, E. Chiaveri ${ }^{7,8}$, M. Chin ${ }^{8}$, G. Cortés ${ }^{11}$, M.A. Cortés-Giraldo ${ }^{13}, \mathrm{M}$. Diakaki ${ }^{14}, \mathrm{C}$. Domingo-Pardo ${ }^{15}$, I. Duran ${ }^{16}$, R. Dressler ${ }^{17}$, C. Eleftheriadis ${ }^{18}$, A. Ferrari ${ }^{8}$, K. Fraval ${ }^{7}$, S. Ganesan $^{19}$, A.R. García ${ }^{5}$, G. Giubrone ${ }^{15}$, I.F. Gonçalves ${ }^{12}$, E. González-Romero ${ }^{5}$, E. Griesmayer ${ }^{20}$, C. Guerrero ${ }^{8}$, F. Gunsing ${ }^{7}$, A. Hernández-Prieto ${ }^{8,11}$, D.G. Jenkins ${ }^{21}$, E. Jericha ${ }^{20}$, Y. Kadi ${ }^{8}$, F. Käppeler ${ }^{22}$, D. Karadimos ${ }^{14}$, N. Kivel ${ }^{17}$, P. Koehler ${ }^{23}$, M. Krtička ${ }^{6}$, J. Kroll ${ }^{6}$, C. Lampoudis ${ }^{7}$, C. Langer ${ }^{2}$, E. Leal-Cidoncha ${ }^{16}$, C. Lederer ${ }^{24}$, H. Leeb ${ }^{20}$, L.S. Leong ${ }^{4}$, R. Losito ${ }^{8}$, A. Manousos ${ }^{18}$, J. Marganiec ${ }^{3}$, T. Martínez $^{5}$, C. Massimi ${ }^{25}$, P.F. Mastinu ${ }^{26}$, M. Mastromarco ${ }^{1}$, E. Mendoza ${ }^{5}$, A. Mengoni ${ }^{27}$, P.M. Milazzo ${ }^{28}$, F. Mingrone ${ }^{25}$, M. Mirea ${ }^{29}$, W. Mondalaers ${ }^{30}$, C. Paradela ${ }^{16}$, A. Pavlik ${ }^{24}$, J. Perkowski ${ }^{3}$, A. Plompen ${ }^{30}$, J. Praena ${ }^{13}$, J.M. Quesada ${ }^{13}$, T. Rauscher ${ }^{31}$, R. Reifarth ${ }^{2}$, A. Riego ${ }^{11}$, C. Rubbia ${ }^{8}$, M. Sabaté-Gilarte ${ }^{13}$, R. Sarmento ${ }^{12}$, A. Saxena ${ }^{19}$, P. Schillebeeckx ${ }^{30}$, S. Schmidt ${ }^{2}$, D. Schumann ${ }^{17}$, G. Tagliente ${ }^{1}$, J.L. Tain ${ }^{15}$, D. Tarrío ${ }^{16}$, L. Tassan-Got ${ }^{4}$, A. Tsinganis ${ }^{8}$, S. Valenta ${ }^{6}$, G. Vannini ${ }^{25}$, V. Variale ${ }^{1}$, P. Vaz ${ }^{12}$, A. Ventura ${ }^{27}$, M.J. Vermeulen $^{21}$, V. Vlachoudis ${ }^{8}$, R. Vlastou ${ }^{14}$,

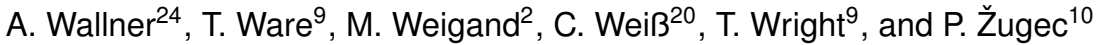

${ }^{1}$ Istituto Nazionale Fisica Nucleare, Sezione di Bari, V. Orabona 4, 70125 Bari, Italy

${ }^{2}$ Johann-Wolfgang-Goethe Universität, Frankfurt, Germany

${ }^{3}$ Uniwersytet Łódzki, Lodz, Poland

${ }^{4}$ Centre National de la Recherche Scientifique/IN2P3 - IPN, Orsay, France

${ }^{5}$ Centro de Investigaciones Energeticas Medioambientales y Tecnológicas (CIEMAT), Madrid, Spain

${ }^{6}$ Charles University, Prague, Czech Republic

${ }^{7}$ Commissariat à l'Énergie Atomique (CEA) Saclay - Irfu, Gif-sur-Yvette, France

${ }^{8}$ European Organization for Nuclear Research (CERN), Geneva, Switzerland

${ }^{9}$ University of Manchester, Oxford Road, Manchester, UK

${ }^{10}$ Department of Physics, Faculty of Science, University of Zagreb, Croatia

${ }^{11}$ Universitat Politecnica de Catalunya, Barcelona, Spain

${ }^{12}$ Instituto Tecnológico e Nuclear, Instituto Superior Técnico, Universidade Técnica de Lisboa, Lisboa, Portugal

${ }^{13}$ Universidad de Sevilla, Spain

${ }^{14}$ National Technical University of Athens (NTUA), Greece

${ }^{15}$ Instituto de Física Corpuscular, CSIC-Universidad de Valencia, Spain

${ }^{16}$ Universidade de Santiago de Compostela, Spain

${ }^{17}$ Paul Scherrer Institut, Villigen PSI, Switzerland

${ }^{18}$ Aristotle University of Thessaloniki, Thessaloniki, Greece

${ }^{19}$ Bhabha Atomic Research Centre (BARC), Mumbai, India

${ }^{20}$ Atominstitut, Technische Universität Wien, Austria

ae-mail: massimo.barbagallo@ba.infn.it

This is an Open Access article distributed under the terms of the Creative Commons Attribution License 2.0, which permits unrestricted use, distribution, and reproduction in any medium, provided the original work is properly cited. 
${ }^{21}$ University of York, Heslington, York, UK

${ }^{22}$ Karlsruhe Institute of Technology, Campus Nord, Institut für Kernphysik, Karlsruhe, Germany

${ }^{23}$ Department of Physics, University of Oslo, N-0316 Oslo, Norway

${ }^{24}$ University of Vienna, Faculty of Physics, Austria

${ }^{25}$ Dipartimento di Fisica, Università di Bologna and Sezione INFN di Bologna, Italy

${ }^{26}$ Istituto Nazionale di Fisica Nucleare, Laboratori Nazionali di Legnaro, Italy

${ }^{27}$ Agenzia nazionale per le nuove tecnologie, l'energia e lo sviluppo economico sostenibile (ENEA), Bologna, Italy

${ }^{28}$ Istituto Nazionale di Fisica Nucleare, Laboratori Nazionali di Trieste, Italy

${ }^{29}$ Horia Hulubei National Institute of Physics and Nuclear Engineering - IFIN HH, Bucharest - Magurele, Romania

${ }^{30}$ European Commission JRC, Institute for Reference Materials and Measurements, B-244 Geel, Belgium

${ }^{31}$ Department of Physics and Astronomy - University of Basel, Basel, Switzerland

\begin{abstract}
The n_TOF facility operates at CERN with the aim of addressing the request of high accuracy nuclear data for advanced nuclear energy systems as well as for nuclear astrophysics. Thanks to the features of the neutron beam, important results have been obtained on neutron induced fission and capture cross sections of $\mathrm{U}, \mathrm{Pu}$ and minor actinides. Recently the construction of another beam line has started; the new line will be complementary to the first one, allowing to further extend the experimental program foreseen for next measurement campaigns.
\end{abstract}

\title{
1 Introduction
}

The development of new systems in nuclear technology has raised the need for high-accuracy neutron cross-section data on a variety of isotopes. At present, available data are insufficient for new medical and industrial applications, and in particular for the development of innovative concepts for safer energy production and nuclear waste incineration, such as Accelerator Driven Systems (ADS), Gen IV fast reactors or reactors based on $\mathrm{Th} / \mathrm{U}$ fuel cycle.

The neutron time-of-flight facility $\mathrm{n}_{-}$TOF was built at CERN more than ten years ago with the aim of providing new and accurate cross sections of neutron-induced reactions of interest for fundamental and applied nuclear science. The experimental activity is focused mostly on the measurement of capture and fission reactions relevant to nuclear technology [1] as well as on neutron capture reactions involved in stellar nucleosynthesis [2]. n_TOF is a white neutron source, where neutrons are produced by spallation of a $20 \mathrm{GeV}$ proton beam on a massive lead block and subsequently moderated by either natural or borated water. The neutron beam in the current experimental area, located at $185 \mathrm{~m}$ from the spallation target, is characterized by a unique combination of high luminosity, wide energy range, high resolution and low duty cycle (Table 1). As a consequences of the high instantaneous flux, the facility is particularly suitable for measurements involving low cross section, radioactive isotopes and samples available in small quantity.

Table 1. Main features of the $n \_T O F$ facility.

\begin{tabular}{lc}
\hline Neutron beam energy range & $27 \mathrm{meV}-1 \mathrm{GeV}$ \\
Proton beam energy and intensity & $20 \mathrm{GeV} ; 7 \times 10^{12} \mathrm{p} /$ pulse \\
Energy resolution & $10^{-4}-10^{-3}$ \\
Repetition rate & $\sim 0.8 \mathrm{~Hz}$ \\
\hline
\end{tabular}




\section{Experimental setup}

Different detection systems have been developed and used by the n_TOF Collaboration for fission and capture cross section measurements as well as for the accurate determination and monitoring of the incident neutron flux [3].

Prompt $\gamma$-rays from the de-excitation of compound nucleus formed in capture reactions are detected with two systems: a pair of low neutron sensitivity $\mathrm{C}_{6} \mathrm{D}_{6}$ liquid scintillator detectors [4] and a Total Absorption Calorimeter (TAC), made of $40 \mathrm{BaF}_{2}$ crystals [5]. The intrinsically low neutron sensitivity of $\mathrm{C}_{6} \mathrm{D}_{6}$ detectors allows to minimize the background induced by scattered neutrons which typically affects capture measurements. The TAC instead is characterized by high geometric and intrinsic efficiency that allow to detect and reconstruct the full $\gamma$-ray cascade from the capture events, thus discriminating them from fission reactions and from various sources of background. However the device is affected by a large neutron sensitivity. For these reasons, accurate cross sections on a given isotope at $n_{-}$TOF are often obtained by combining results from the two complementary detection systems.

Fission cross-section measurements are routinely carried out with different detector systems. In the past Fission Ionization Chambers (FIC) [6] have been used. More recently, the FICs have been replaced with high performance MicroMegas detectors [7]. The detector is composed of a gas volume separated into two small regions by a thin micromesh; it has several advantages, such as high radiation hardness, a high signal-to-noise ratio and a good discrimination of $\alpha$-particles from fission fragments. Another method used at $\mathrm{n}_{-}$TOF relies on the detection of both fission fragments in coincidence, by means of a stack of position-sensitive Parallel Plate Avalanche Counters (PPACs) [8], with the fissile sample placed in between two detectors. The position sensitivity of the device allows to measure, together with the cross-section, the angular distribution of fission fragments.

\section{Results and perspectives}

The accuracy of neutron cross-sections for a great number of isotopes, many of which of interest for nuclear technologies, has been improved thanks to data collected at n_TOF during the two experimental campaigns performed so far (2001-2004 and 2009-2012). Some of the most important results obtained are mentioned hereafter.

High accuracy results have been obtained on the radiative capture of ${ }^{232} \mathrm{Th}$ and fission of ${ }^{233} \mathrm{U}$, both of fundamental importance for the development of the Th/U fuel cycle: ${ }^{232} \mathrm{Th}(\mathrm{n}, \gamma)$ cross section has been determined from $1 \mathrm{eV}$ to $1 \mathrm{MeV}$ with a 5\% accuracy $[9,10]$ and in the Unresolved Resonances Region (URR) a long standing discrepancy of $40 \%$ between previous data has been finally solved; similarly high quality results have been obtained on ${ }^{233} \mathrm{U}(\mathrm{n}, \mathrm{f})[11,12]$ from thermal to 20 $\mathrm{MeV}$. High accuracy fission cross-sections have been obtained also on ${ }^{237} \mathrm{~Np}$ and ${ }^{240} \mathrm{Pu}[13,14]$, two of the minor actinides most abundantly produced in current reactors. These data are needed in transmutation projects or for the design of Gen IV fast nuclear reactors. Recently capture cross section of ${ }^{238} \mathrm{U},{ }^{236} \mathrm{U},{ }^{241} \mathrm{Am}$ and fission cross section of ${ }^{240} \mathrm{Pu}$ and ${ }^{242} \mathrm{Pu}$ have been measured with the aim of reaching unprecedented accuracy.

In Figure 1 preliminary results on the radiative capture cross section of ${ }^{236} \mathrm{U}$ measured at $\mathrm{n}_{-} \mathrm{TOF}$ with two detection systems are shown and compared with ENDF/B-VII.0 evaluation or previous experimental data. At $n \_$TOF ${ }^{236} \mathrm{U}(\mathrm{n}, \gamma)$ cross-section has been measured for the first time in the whole energy region from thermal energy up to $1 \mathrm{MeV}$. In the Resolved Resonance Region (RRR), the n_TOF results indicate that the evaluated library need some refinement on both the strength and energy of the resonances (left panel), while in the Unresolved Resonance Region (URR) the new data finally rule out some previous results, confirming current evaluations (right panel). 

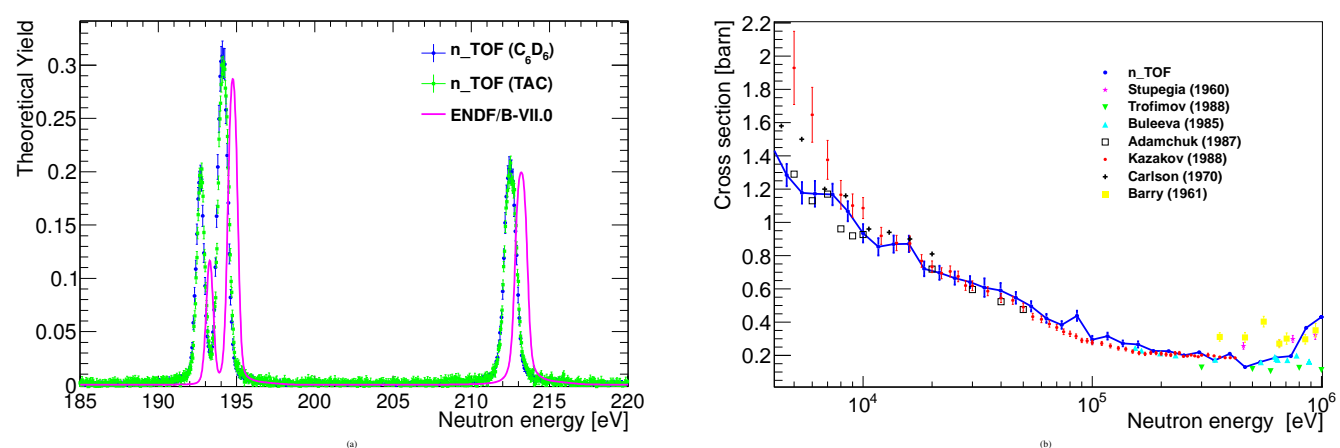

Figure 1. The $n \_$TOF results with both capture setups for ${ }^{236} U(n, \gamma)$ cross section measurement in comparison with ENDF/B-VII.0 evaluation in RRR (left panel) and with previous experimental data in URR (right panel).

To expand the potentiality of the facility a new beam line and corresponding experimental area are being constructed and will be completed in May 2014. The shorter flight path of $20 \mathrm{~m}$ will ensure a 25 times higher neutron flux in this new experimental area and a factor 250 in the signal/background ratio. Although with a loss in energy resolution (due to the shorter flight path), the extremely high instantaneous neutron flux will allow to perform a new set of measurements which are not feasible at the moment, particularly those on radioactive isotopes with lifetime as short as a few years.

A rich experimental program is foreseen at $n \_$TOF for the next years, both in the far (200 m, EAR1) and in the near (20 m, EAR2) experimental areas. Among them several measurements are being planned of interest for Nuclear Astrophysics, Nuclear Medicine and Basic Nuclear Physics. New measurements of interest for nuclear technology are foreseen in EAR1, such as the simultaneous capture and fission cross section of ${ }^{233} \mathrm{U}$, the fission of ${ }^{231} \mathrm{~Pa}$ and capture of ${ }^{242} \mathrm{Pu}$, while in the new experimental area the Collaboration is planning challenging measurements such as ${ }^{238,241} \mathrm{Pu}(\mathrm{n}, \mathrm{f}),{ }^{244} \mathrm{Cm}(\mathrm{n}, \mathrm{f})$, ${ }^{232} \mathrm{U}(\mathrm{n}, \mathrm{f})$ and ${ }^{245} \mathrm{Cm}(\mathrm{n}, \gamma)$.

\section{References}

[1] N.Colonna et al., Energy Environ. Sci. 3, 157 (2010) 1910-1917

[2] F. Käppeler, R. Gallino, S. Bisterzo, and Wako Aoki, Rev. Mod. Phys. 83, 157 (2011)

[3] M. Barbagallo et al., Submitted to Eur. Phys. J. A.

[4] R. Plag et al., Nucl. Instr. And Meth. A. 496, 425-436 (2003).

[5] C. Guerrero et al., AIP Conf. 1090, 372-375 (2008).

[6] M. Calviani et al., Nucl. Instr. And Meth. A. 594, 220 (2008).

[7] I. Giomataris and R. De Oliveira, Patent CEA-CERN, Application Number 09290825.0 (2009).

[8] C. Paradela et al., Phys. Rev. C 82, (2010) 044604.

[9] F. Gunsing et al., Phys. Rev. C 85, (2012) 064601.

[10] G. Aerts et al., Phys. Rev. C 73, (2006) 054610.

[11] M. Calviani et al., Phys. Rev. C 80, (2009) 044604.

[12] F. Belloni et al., Eur. Phys. J. A. 47, 2 (2011).

[13] C. Paradela et al., Phys. Rev. C 82, (2010) 034601.

[14] C. Guerrero et al., Phys. Rev. C 85, (2012) 044616. 